\title{
On 5-regular bipartitions with even parts distinct
}

\author{
M. S. Mahadeva Naika1 ${ }^{1}$ T. Harishkumar ${ }^{1}$
}

Received: 21 February 2018 / Accepted: 21 May 2018 / Published online: 4 September 2018

C Springer Science+Business Media, LLC, part of Springer Nature 2018

\begin{abstract}
In 2010, Andrews, Michael D. Hirschhorn and James A. Sellers considered the function ped $(n)$, the number of partition of an integer $n$ with even parts distinct (the odd parts are unrestricted). They obtained infinite families of congruences in the spirit of Ramanujan's congruences for the unrestricted partition function $p(n)$. Let $b(n)$ denote the number of 5-regular bipartitions of a positive integer $n$ with even parts distinct (odd parts are unrestricted). In this paper, we establish many infinite families of congruences modulo powers of 2 for $b(n)$. For example,
\end{abstract}

$$
\sum_{n=0}^{\infty} b\left(16 \cdot 3^{2 \alpha} \cdot 5^{2 \beta} n+14 \cdot 3^{2 \alpha} \cdot 5^{2 \beta}+1\right) q^{n} \equiv 8 f_{2}^{3} f_{5}^{3} \quad(\bmod 16)
$$

where $\alpha, \beta \geq 0$.

Keywords Partition identities · Theta-functions · Partition congruences - Regular bipartition

Mathematics Subject Classification 11P83 - 05A17

\section{Introduction}

A partition of a positive integer $n$ is a non-increasing sequence of positive integers whose sum is $n$. An $\ell$-regular partition is a partition in which none of the parts is

M. S. Mahadeva Naika

msmnaika@rediffmail.com

T. Harishkumar

harishhaf@gmail.com

1 Department of Mathematics, Central College Campus, Bangalore University, Bengaluru, Karnataka, India 
divisible by $\ell$. Let $b_{\ell}(n)$ denote the number of $\ell$-regular partitions of $n$ with $b_{\ell}(0)=1$. The generating function for $b_{\ell}(n)$ is

$$
\sum_{n=0}^{\infty} b_{\ell}(n) q^{n}=\frac{f_{\ell}}{f_{1}}
$$

where $f_{\ell}:=\left(q^{\ell} ; q^{\ell}\right)_{\infty}=\prod_{n=1}^{\infty}\left(1-q^{n \ell}\right)$.

Arithmetic properties of $\ell$-regular partition functions have been studied by a number of mathematicians. Calkin et al. [3] established congruences for 5-regular partitions modulo 2 and for 13-regular partitions modulo 2 and 3 using the theory of modular forms. For more details, one can see [5,7,9].

In 2010, Andrews et al. [1] obtained infinite families of congruences for ped $(n)$, the number of partitions of $n$ with even parts distinct. For more details one can see $[4,11]$.

Recently, Lin [8] proved two infinite families of congruences modulo 3 for Bped $(n)$, the number of bipartitions of $n$ with even parts distinct.

Let $b(n)$ denote the number of 5-regular bipartitions of $n$ with even parts distinct with $b(0)=1$. The generating function is given by

$$
\sum_{n=0}^{\infty} b(n) q^{n}=\frac{f_{4}^{2} f_{5}^{2}}{f_{1}^{2} f_{20}^{2}}
$$

We prove many congruences of the following form. For $\alpha, \beta \geq 0$,

$$
\sum_{n=0}^{\infty} b\left(16 \cdot 3^{2 \alpha} \cdot 5^{2 \beta} n+14 \cdot 3^{2 \alpha} \cdot 5^{2 \beta}+1\right) q^{n} \equiv 8 f_{2}^{3} f_{5}^{3} \quad(\bmod 16)
$$

\section{Preliminary results}

In this section, we record several identities which are useful in proving our main results.

Lemma 2.1 The following 2-dissections hold:

$$
\frac{1}{f_{1}^{2}}=\frac{f_{8}^{5}}{f_{2}^{5} f_{16}^{2}}+2 q \frac{f_{4}^{2} f_{16}^{2}}{f_{2}^{5} f_{8}}
$$

and

$$
\frac{1}{f_{1}^{4}}=\frac{f_{4}^{14}}{f_{2}^{14} f_{8}^{4}}+4 q \frac{f_{4}^{2} f_{8}^{4}}{f_{2}^{10}}
$$

For proofs, see [2, p. 40]. 
Lemma 2.2 We have

$$
f_{1}^{3}=\frac{f_{6} f_{9}^{6}}{f_{3} f_{18}^{3}}-3 q f_{9}^{3}+4 q^{3} \frac{f_{3}^{2} f_{18}^{6}}{f_{6}^{2} f_{9}^{3}}
$$

For a proof, see [2, p. 345].

Lemma 2.3 The following 2-dissections hold:

$$
\frac{f_{5}}{f_{1}}=\frac{f_{8} f_{20}^{2}}{f_{2}^{2} f_{40}}+q \frac{f_{4}^{3} f_{10} f_{40}}{f_{2}^{3} f_{8} f_{20}}
$$

and

$$
\frac{f_{1}}{f_{5}}=\frac{f_{2} f_{8} f_{20}^{3}}{f_{4} f_{10}^{3} f_{40}}-q \frac{f_{4}^{2} f_{40}}{f_{8} f_{10}^{2}}
$$

For proofs, see [7].

Lemma 2.4 We have

$$
\begin{aligned}
& \frac{1}{f_{1}^{3} f_{5}}=\frac{f_{4}^{4}}{f_{2}^{7} f_{10}}-2 q \frac{f_{4}^{6} f_{20}^{2}}{f_{2}^{9} f_{10}^{3}}+5 q \frac{f_{4}^{3} f_{20}}{f_{2}^{8}}+2 q^{2} \frac{f_{4}^{9} f_{40}^{2}}{f_{2}^{10} f_{8}^{2} f_{10}^{2} f_{20}} \\
& f_{1}^{3} f_{5}=\frac{f_{2}^{2} f_{4} f_{10}^{2}}{f_{20}}+2 q f_{4}^{3} f_{20}-5 q f_{2} f_{10}^{3}+2 q^{2} \frac{f_{4}^{6} f_{10} f_{40}^{2}}{f_{2} f_{8}^{2} f_{20}^{2}}
\end{aligned}
$$

and

$$
f_{1} f_{5}^{3}=f_{2}^{3} f_{10}-q \frac{f_{2}^{2} f_{10}^{2} f_{20}}{f_{4}}+2 q^{2} f_{4} f_{20}^{3}-2 q^{3} \frac{f_{4}^{4} f_{10} f_{40}^{2}}{f_{2} f_{8}^{2}} .
$$

For proofs, see [10].

Lemma 2.5 [2, p. 303, Entry 17(v)] We have

$$
(q ; q)_{\infty}=\left(q^{49} ; q^{49}\right)_{\infty}\left(\frac{B\left(q^{7}\right)}{C\left(q^{7}\right)}-q \frac{A\left(q^{7}\right)}{B\left(q^{7}\right)}-q^{2}+q^{5} \frac{C\left(q^{7}\right)}{A\left(q^{7}\right)}\right),
$$

where $A(q)=f\left(-q^{3},-q^{4}\right), B(q)=f\left(-q^{2},-q^{5}\right)$ and $C(q)=f\left(-q,-q^{6}\right)$.

We prove the following theorem,

Theorem 2.1 Let $r_{1} \in\{62,78\}, r_{2} \in\{62,158\}, r_{3} \in\{166,214\}, r_{4} \in\{142,238\}$, $r_{5} \in\{86,134\}, r_{6} \in\{10,26\}, r_{7} \in\{28,92,124,156\}, r_{8} \in\{124,156\}, r_{9} \in\{22,38\}$, $r_{10} \in\{34,66\}, r_{11} \in\{26,42,58,74\}, r_{12} \in\{44,76\}, r_{13} \in\{68,132\}$ and $r_{14} \in$ $\{52,84,116,148\}$. Then for $\alpha, \beta, \gamma \geq 0$, we have, modulo 16,

$$
\sum_{n=0}^{\infty} b\left(16 \cdot 5^{2 \beta} n+6 \cdot 5^{2 \beta}+1\right) q^{n} \equiv 8 f_{1}^{9}+8 f_{4} f_{5},
$$


$\sum_{n=0}^{\infty} b\left(16 \cdot 5^{2 \beta+1} n+14 \cdot 5^{2 \beta+1}+1\right) q^{n} \equiv f_{1} f_{20}+8 q f_{5}^{9}$,

$b\left(16 \cdot 5^{2 \beta+2} n+r_{1} \cdot 5^{2 \beta+1}+1\right) \equiv 0$,

$\sum_{n=0}^{\infty} b\left(16 \cdot 3^{2 \alpha} \cdot 5^{2 \beta} n+14 \cdot 3^{2 \alpha} \cdot 5^{2 \beta}+1\right) q^{n} \equiv 8 f_{2}^{3} f_{5}^{3}$,

$\sum_{n=0}^{\infty} b\left(16 \cdot 3^{2 \alpha} \cdot 5^{2 \beta+1} n+6 \cdot 3^{2 \alpha} \cdot 5^{2 \beta+1}+1\right) q^{n} \equiv 8 q f_{1}^{3} f_{10}^{3}$,

$\sum_{n=0}^{\infty} b\left(16 \cdot 3^{2 \alpha+1} \cdot 5^{2 \beta} n+14 \cdot 3^{2 \alpha} \cdot 5^{2 \beta}+1\right) q^{n} \equiv 8 f_{2} f_{5}$,

$\sum_{n=0}^{\infty} b\left(16 \cdot 3^{2 \alpha+1} \cdot 5^{2 \beta+1} n+22 \cdot 3^{2 \alpha} \cdot 5^{2 \beta+1}+1\right) q^{n} \equiv 8 f_{1} f_{10}$,

$\sum_{n=0}^{\infty} b\left(16 \cdot 3^{2 \alpha+1} \cdot 5^{2 \beta} n+2 \cdot 3^{2 \alpha+1} \cdot 5^{2 \beta+1}+1\right) q^{n} \equiv 8 q^{2} f_{6}^{3} f_{15}^{3}$,

$\sum_{n=0}^{\infty} b\left(16 \cdot 3^{2 \alpha+1} \cdot 5^{2 \beta} n+46 \cdot 3^{2 \alpha} \cdot 5^{2 \beta}+1\right) q^{n} \equiv 8 f_{5} f_{6}^{3}+8 q f_{2} f_{15}^{3}$,

$\sum_{n=0}^{\infty} b\left(16 \cdot 3^{2 \alpha+1} \cdot 5^{2 \beta+1} n+38 \cdot 3^{2 \alpha} \cdot 5^{2 \beta+1}+1\right) q^{n} \equiv 8 f_{3}^{3} f_{10}+8 q^{3} f_{1} f_{30}^{3}$

$b\left(16 \cdot 3^{2 \alpha+1} \cdot 5^{2 \beta+1} n+r_{2} \cdot 3^{2 \alpha} \cdot 5^{2 \beta}+1\right) \equiv 0$,

$b\left(16 \cdot 3^{2 \alpha+1} \cdot 5^{2 \beta+2} n+r_{3} \cdot 3^{2 \alpha} \cdot 5^{2 \beta+1}+1\right) \equiv 0$,

$b\left(16 \cdot 3^{2 \alpha+1} \cdot 5^{2 \beta+1} n+r_{4} \cdot 3^{2 \alpha} \cdot 5^{2 \beta}+1\right) \equiv 0$,

$b\left(16 \cdot 3^{2 \alpha+1} \cdot 5^{2 \beta+2} n+r_{5} \cdot 3^{2 \alpha} \cdot 5^{2 \beta+1}+1\right) \equiv 0$,

$b\left(16 \cdot 3^{2 \alpha+2} \cdot 5^{2 \beta} n+r_{6} \cdot 3^{2 \alpha+1} \cdot 5^{2 \beta}+1\right) \equiv 0$,

$\sum_{n=0}^{\infty} b\left(32 \cdot 3^{4 \alpha} \cdot 5^{2 \beta} \cdot 7^{2 \gamma} n+12 \cdot 3^{4 \alpha} \cdot 5^{2 \beta} \cdot 7^{2 \gamma}+1\right) q^{n} \equiv 8 f_{1}^{9}$,

$\sum_{n=0}^{\infty} b\left(32 \cdot 3^{4 \alpha} \cdot 5^{2 \beta} \cdot 7^{2 \gamma+1} n+4 \cdot 3^{4 \alpha} \cdot 5^{2 \beta+1} \cdot 7^{2 \gamma+1}+1\right) q^{n} \equiv 8 q^{2} f_{7}^{9}$,

$\sum_{n=0}^{\infty} b\left(32 \cdot 3^{4 \alpha} \cdot 5^{2 \beta+1} \cdot 7^{2 \gamma} n+4 \cdot 3^{4 \alpha} \cdot 5^{2 \beta+1} \cdot 7^{2 \gamma+1}+1\right) q^{n} \equiv 8 q f_{5}^{9}$, 


$$
\begin{aligned}
& b\left(32 \cdot 3^{4 \alpha} \cdot 5^{2 \beta+2} \cdot 7^{2 \gamma} n+r_{7} \cdot 3^{4 \alpha} \cdot 5^{2 \beta+1} \cdot 7^{2 \gamma}+1\right) \equiv 0, \\
& \sum_{n=0}^{\infty} b\left(32 \cdot 3^{4 \alpha+1} \cdot 5^{2 \beta} \cdot 7^{2 \gamma} n+44 \cdot 3^{4 \alpha} \cdot 5^{2 \beta} \cdot 7^{2 \gamma}+1\right) q^{n} \equiv 8 f_{2} f_{3}^{3} \text {, } \\
& \sum_{n=0}^{\infty} b\left(32 \cdot 3^{4 \alpha+1} \cdot 5^{2 \beta} \cdot 7^{2 \gamma} n+76 \cdot 3^{4 \alpha} \cdot 5^{2 \beta} \cdot 7^{2 \gamma}+1\right) q^{n} \equiv 8 f_{1} f_{6}^{3}, \\
& \sum_{n=0}^{\infty} b\left(32 \cdot 3^{4 \alpha+1} \cdot 5^{2 \beta+1} \cdot 7^{2 \gamma} n+4 \cdot 3^{4 \alpha} \cdot 5^{2 \beta+1} \cdot 7^{2 \gamma+1}+1\right) q^{n} \\
& \equiv 8 q^{2} f_{10} f_{15}^{3}, \\
& \sum_{n=0}^{\infty} b\left(32 \cdot 3^{4 \alpha+1} \cdot 5^{2 \beta+1} \cdot 7^{2 \gamma} n+92 \cdot 3^{4 \alpha} \cdot 5^{2 \beta+1} \cdot 7^{2 \gamma}+1\right) q^{n} \\
& \equiv 8 q^{3} f_{5} f_{30}^{3}, \\
& \sum_{n=0}^{\infty} b\left(32 \cdot 5^{2 \beta} n+28 \cdot 5^{2 \beta}+1\right) q^{n} \equiv 8 f_{1} f_{20}+8 f_{2}^{3} f_{5}^{3}, \\
& \sum_{n=0}^{\infty} b\left(32 \cdot 5^{2 \beta+1} n+12 \cdot 5^{2 \beta+1}+1\right) q^{n} \equiv 8 f_{4} f_{5}+8 q f_{1}^{3} f_{10}^{3}, \\
& b\left(32 \cdot 5^{2 \beta+1} n+r_{8} \cdot 5^{2 \beta}+1\right) \equiv 0
\end{aligned}
$$

and modulo 4,

$$
\begin{aligned}
& \sum_{n=0}^{\infty} b\left(16 \cdot 3^{2 \alpha} \cdot 5^{2 \beta} \cdot 7^{2 \gamma} n+2 \cdot 3^{2 \alpha} \cdot 5^{2 \beta} \cdot 7^{2 \gamma}+1\right) q^{n} \equiv 2 f_{1}^{3}, \\
& \sum_{n=0}^{\infty} b\left(16 \cdot 3^{2 \alpha} \cdot 5^{2 \beta} \cdot 7^{2 \gamma+1} n+2 \cdot 3^{2 \alpha} \cdot 5^{2 \beta} \cdot 7^{2 \gamma+2}+1\right) q^{n} \equiv 2 f_{7}^{3}, \\
& b\left(16 \cdot 3^{2 \alpha+1} \cdot 5^{2 \beta} \cdot 7^{2 \gamma} n+2 \cdot 3^{2 \alpha} \cdot 5^{2 \beta} \cdot 7^{2 \gamma}+1\right) \\
& \equiv\left\{\begin{array}{l}
2 \text { if } n=k(3 k+1) / 2 \text { for some } k \in \mathbb{Z}, \\
0 \text { otherwise. }
\end{array}\right. \\
& \sum_{n=0}^{\infty} b\left(16 \cdot 3^{2 \alpha+1} \cdot 5^{2 \beta} \cdot 7^{2 \gamma} n+6 \cdot 3^{2 \alpha+1} \cdot 5^{2 \beta} \cdot 7^{2 \gamma}+1\right) q^{n} \equiv 2 f_{3}^{3}, \\
& b\left(16 \cdot 3^{2 \alpha+1} \cdot 5^{2 \beta} \cdot 7^{2 \gamma} n+34 \cdot 3^{2 \alpha} \cdot 5^{2 \beta} \cdot 7^{2 \gamma}+1\right) \equiv 0, \\
& b\left(16 \cdot 3^{2 \alpha+2} \cdot 5^{2 \beta} \cdot 7^{2 \gamma} n+r_{9} \cdot 3^{2 \alpha+1} \cdot 5^{2 \beta} \cdot 7^{2 \gamma}+1\right) \equiv 0, \\
& \sum_{n=0}^{\infty} b\left(16 \cdot 3^{2 \alpha} \cdot 5^{2 \beta+1} \cdot 7^{2 \gamma} n+2 \cdot 3^{2 \alpha} \cdot 5^{2 \beta+2} \cdot 7^{2 \gamma}+1\right) q^{n} \equiv 2 f_{5}^{3},
\end{aligned}
$$




$$
\begin{aligned}
& b\left(16 \cdot 3^{2 \alpha} \cdot 5^{2 \beta+1} \cdot 7^{2 \gamma} n+r_{10} \cdot 3^{2 \alpha} \cdot 5^{2 \beta} \cdot 7^{2 \gamma}+1\right) \equiv 0, \\
& b\left(16 \cdot 3^{2 \alpha} \cdot 5^{2 \beta+2} \cdot 7^{2 \gamma} n+r_{11} \cdot 3^{2 \alpha} \cdot 5^{2 \beta+1} \cdot 7^{2 \gamma}+1\right) \equiv 0, \\
& \sum_{n=0}^{\infty} b\left(16 \cdot 3^{2 \alpha} \cdot 5^{2 \beta+1} \cdot 7^{2 \gamma} n+2 \cdot 3^{2 \alpha} \cdot 5^{2 \beta+1} \cdot 7^{2 \gamma}+1\right) q^{n} \equiv 2 f_{1}^{3}, \\
& \sum_{n=0}^{\infty} b\left(16 \cdot 3^{2 \alpha} \cdot 5^{2 \beta+1} \cdot 7^{2 \gamma+1} n+2 \cdot 3^{2 \alpha} \cdot 5^{2 \beta+1} \cdot 7^{2 \gamma+2}+1\right) q^{n} \equiv 2 f_{7}^{3}, \\
& b\left(16 \cdot 3^{2 \alpha+1} \cdot 5^{2 \beta+1} \cdot 7^{2 \gamma} n+2 \cdot 3^{2 \alpha} \cdot 5^{2 \beta+1} \cdot 7^{2 \gamma}+1\right) \\
& \equiv\left\{\begin{array}{l}
2 \text { ifn } n=k(3 k+1) / 2 \text { for some } k \in \mathbb{Z}, \\
0 \text { otherwise. }
\end{array}\right.
\end{aligned}
$$

$\sum_{n=0}^{\infty} b\left(16 \cdot 3^{2 \alpha+1} \cdot 5^{2 \beta+1} \cdot 7^{2 \gamma} n+6 \cdot 3^{2 \alpha+1} \cdot 5^{2 \beta+1} \cdot 7^{2 \gamma}+1\right) q^{n} \equiv 2 f_{3}^{3}$,

$b\left(16 \cdot 3^{2 \alpha+1} \cdot 5^{2 \beta+1} \cdot 7^{2 \gamma} n+34 \cdot 3^{2 \alpha} \cdot 5^{2 \beta+1} \cdot 7^{2 \gamma}+1\right) \equiv 0$,

$b\left(16 \cdot 3^{2 \alpha+2} \cdot 5^{2 \beta+1} \cdot 7^{2 \gamma} n+r_{9} \cdot 3^{2 \alpha+1} \cdot 5^{2 \beta+1} \cdot 7^{2 \gamma}+1\right) \equiv 0$,

$\sum_{n=0}^{\infty} b\left(16 \cdot 3^{2 \alpha} \cdot 5^{2 \beta+2} \cdot 7^{2 \gamma} n+2 \cdot 3^{2 \alpha} \cdot 5^{2 \beta+3} \cdot 7^{2 \gamma}+1\right) q^{n} \equiv 2 f_{5}^{3}$,

$b\left(16 \cdot 3^{2 \alpha} \cdot 5^{2 \beta+2} \cdot 7^{2 \gamma} n+r_{10} \cdot 3^{2 \alpha} \cdot 5^{2 \beta+1} \cdot 7^{2 \gamma}+1\right) \equiv 0$,

$b\left(16 \cdot 3^{2 \alpha} \cdot 5^{2 \beta+3} \cdot 7^{2 \gamma} n+r_{11} \cdot 3^{2 \alpha} \cdot 5^{2 \beta+2} \cdot 7^{2 \gamma}+1\right) \equiv 0$,

$\sum_{n=0}^{\infty} b\left(32 \cdot 3^{2 \alpha} \cdot 5^{2 \beta} \cdot 7^{2 \gamma} n+4 \cdot 3^{2 \alpha} \cdot 5^{2 \beta} \cdot 7^{2 \gamma}+1\right) q^{n} \equiv 2 f_{1}^{3}$,

$\sum_{n=0}^{\infty} b\left(32 \cdot 3^{2 \alpha} \cdot 5^{2 \beta} \cdot 7^{2 \gamma+1} n+4 \cdot 3^{2 \alpha} \cdot 5^{2 \beta} \cdot 7^{2 \gamma+2}+1\right) q^{n} \equiv 2 f_{7}^{3}$,

$b\left(32 \cdot 3^{2 \alpha+1} \cdot 5^{2 \beta} \cdot 7^{2 \gamma} n+4 \cdot 3^{2 \alpha} \cdot 5^{2 \beta} \cdot 7^{2 \gamma}+1\right)$

$$
\equiv\left\{\begin{array}{l}
2 \text { if } n=k(3 k+1) / 2 \text { for some } k \in \mathbb{Z}, \\
0 \text { otherwise. }
\end{array}\right.
$$

$\sum_{n=0}^{\infty} b\left(32 \cdot 3^{2 \alpha+1} \cdot 5^{2 \beta} \cdot 7^{2 \gamma} n+12 \cdot 3^{2 \alpha+1} \cdot 5^{2 \beta} \cdot 7^{2 \gamma}+1\right) q^{n} \equiv 2 f_{3}^{3}$,

$b\left(32 \cdot 3^{2 \alpha+1} \cdot 5^{2 \beta} \cdot 7^{2 \gamma} n+68 \cdot 3^{2 \alpha} \cdot 5^{2 \beta} \cdot 7^{2 \gamma}+1\right) \equiv 0$,

$b\left(32 \cdot 3^{2 \alpha+2} \cdot 5^{2 \beta} \cdot 7^{2 \gamma} n+r_{12} \cdot 3^{2 \alpha+1} \cdot 5^{2 \beta} \cdot 7^{2 \gamma}+1\right) \equiv 0$,

$\sum_{n=0}^{\infty} b\left(32 \cdot 3^{2 \alpha} \cdot 5^{2 \beta+1} \cdot 7^{2 \gamma} n+4 \cdot 3^{2 \alpha} \cdot 5^{2 \beta+2} \cdot 7^{2 \gamma}+1\right) q^{n} \equiv 2 f_{5}^{3}$, 


$$
\begin{aligned}
& b\left(32 \cdot 3^{2 \alpha} \cdot 5^{2 \beta+1} \cdot 7^{2 \gamma} n+r_{13} \cdot 3^{2 \alpha} \cdot 5^{2 \beta} \cdot 7^{2 \gamma}+1\right) \equiv 0, \\
& b\left(32 \cdot 3^{2 \alpha} \cdot 5^{2 \beta+2} \cdot 7^{2 \gamma} n+r_{14} \cdot 3^{2 \alpha} \cdot 5^{2 \beta+1} \cdot 7^{2 \gamma}+1\right) \equiv 0, \\
& \sum_{n=0}^{\infty} b\left(32 \cdot 3^{2 \alpha} \cdot 5^{2 \beta+1} \cdot 7^{2 \gamma} n+4 \cdot 3^{2 \alpha} \cdot 5^{2 \beta+1} \cdot 7^{2 \gamma}+1\right) q^{n} \equiv 2 f_{1}^{3} \text {, } \\
& \sum_{n=0}^{\infty} b\left(32 \cdot 3^{2 \alpha} \cdot 5^{2 \beta+1} \cdot 7^{2 \gamma+1} n+4 \cdot 3^{2 \alpha} \cdot 5^{2 \beta+1} \cdot 7^{2 \gamma+2}+1\right) q^{n} \equiv 2 f_{7}^{3} \text {, } \\
& b\left(32 \cdot 3^{2 \alpha+1} \cdot 5^{2 \beta+1} \cdot 7^{2 \gamma} n+4 \cdot 3^{2 \alpha} \cdot 5^{2 \beta+1} \cdot 7^{2 \gamma}+1\right) \\
& \equiv\left\{\begin{array}{l}
2 \text { if } n=k(3 k+1) / 2 \text { for some } k \in \mathbb{Z}, \\
0 \text { otherwise. }
\end{array}\right. \\
& \sum_{n=0}^{\infty} b\left(32 \cdot 3^{2 \alpha+1} \cdot 5^{2 \beta+1} \cdot 7^{2 \gamma} n+12 \cdot 3^{2 \alpha+1} \cdot 5^{2 \beta+1} \cdot 7^{2 \gamma}+1\right) q^{n} \equiv 2 f_{3}^{3} \text {, } \\
& b\left(32 \cdot 3^{2 \alpha+1} \cdot 5^{2 \beta+1} \cdot 7^{2 \gamma} n+68 \cdot 3^{2 \alpha} \cdot 5^{2 \beta+1} \cdot 7^{2 \gamma}+1\right) \equiv 0, \\
& b\left(32 \cdot 3^{2 \alpha+2} \cdot 5^{2 \beta+1} \cdot 7^{2 \gamma} n+r_{12} \cdot 3^{2 \alpha+1} \cdot 5^{2 \beta+1} \cdot 7^{2 \gamma}+1\right) \equiv 0, \\
& \sum_{n=0}^{\infty} b\left(32 \cdot 3^{2 \alpha} \cdot 5^{2 \beta+2} \cdot 7^{2 \gamma} n+4 \cdot 3^{2 \alpha} \cdot 5^{2 \beta+3} \cdot 7^{2 \gamma}+1\right) q^{n} \equiv 2 f_{5}^{3} \text {, } \\
& b\left(32 \cdot 3^{2 \alpha} \cdot 5^{2 \beta+2} \cdot 7^{2 \gamma} n+r_{13} \cdot 3^{2 \alpha} \cdot 5^{2 \beta+1} \cdot 7^{2 \gamma}+1\right) \equiv 0, \\
& b\left(32 \cdot 3^{2 \alpha} \cdot 5^{2 \beta+3} \cdot 7^{2 \gamma} n+r_{14} \cdot 3^{2 \alpha} \cdot 5^{2 \beta+2} \cdot 7^{2 \gamma}+1\right) \equiv 0 .
\end{aligned}
$$

\section{Proof of the Theorem (2.1)}

Using (2.4) in (1.1) and comparing the terms involving $q^{2 n+1}$ from both sides, we arrive at

$$
\sum_{n=0}^{\infty} b(2 n+1) q^{n}=2 \frac{f_{2}^{5} f_{5}}{f_{1}^{5} f_{10}} .
$$

From the binomial theorem, it is easy to see that for any positive integers $k$ and $m$,

$$
\begin{aligned}
f_{k}^{2 m} & \equiv f_{2 k}^{m} \quad(\bmod 2), \\
f_{k}^{4 m} & \equiv f_{2 k}^{2 m} \quad(\bmod 4), \\
f_{k}^{8 m} & \equiv f_{2 k}^{4 m} \quad(\bmod 8) .
\end{aligned}
$$

Employing (2.2) and (2.4) along with (3.2) and (3.4) in (3.1), we get, modulo 16,

$$
\sum_{n=0}^{\infty} b(4 n+1) q^{n} \equiv 2 \frac{f_{2}^{2} f_{4} f_{10}^{2}}{f_{1}^{3} f_{5} f_{20}}+8 q f_{2}^{7} f_{10},
$$




$$
\sum_{n=0}^{\infty} b(4 n+3) q^{n} \equiv 2 \frac{f_{2}^{5} f_{20}}{f_{1}^{4} f_{4} f_{10}}+8 \frac{f_{4}^{5}}{f_{1}^{3} f_{5}}
$$

Utilizing (2.2) and (2.6) in (3.6), we arrive at

$$
\sum_{n=0}^{\infty} b(8 n+3) q^{n} \equiv 2 \frac{f_{2} f_{10}}{f_{1} f_{5}}+8 \frac{f_{2}^{7}}{f_{1}^{3} f_{5}}
$$

and

$$
\sum_{n=0}^{\infty} b(8 n+7) q^{n} \equiv 8 f_{8} f_{10}+8 f_{1}^{3} f_{2}^{5} f_{5} .
$$

Using (2.7) in (3.8), we get

$$
\sum_{n=0}^{\infty} b(16 n+7) q^{n} \equiv 8 f_{1}^{9}+8 f_{4} f_{5}
$$

and

$$
\sum_{n=0}^{\infty} b(16 n+15) q^{n} \equiv 8 f_{2}^{3} f_{5}^{3} .
$$

The congruence (3.9) is $\beta=0$ case of (2.10). Suppose that the congruence (2.10) is true for some integer $\beta \geq 0$.

Ramanujan recorded the following identity in his notebooks without proof:

$$
f_{1}=f_{25}\left(R\left(q^{5}\right)^{-1}-q-q^{2} R\left(q^{5}\right)\right)
$$

where $R(q)=\frac{f\left(-q,-q^{4}\right)}{f\left(-q^{2},-q^{3}\right)}$.

For a proof of $(3.11)$, one can see $[6,12]$.

Using (3.11) in (2.10) and then comparing the coefficients of $q^{5 n+4}$, we get

$$
\sum_{n=0}^{\infty} b\left(16 \cdot 5^{2 \beta+1} n+14 \cdot 5^{2 \beta+1}+1\right) q^{n} \equiv 8 f_{1} f_{20}+8 q f_{5}^{9}
$$

which proves (2.11). Again using (3.11) in (3.12) and then comparing the coefficients of $q^{5 n+1}$ in the resultant equation, we obtain

$$
\sum_{n=0}^{\infty} b\left(16 \cdot 5^{2 \beta+2} n+6 \cdot 5^{2 \beta+2}+1\right) q^{n} \equiv 8 f_{1}^{9}+8 f_{4} f_{5}
$$

which implies that the congruence (2.10) is true for $\beta+1$. By mathematical induction, the congruence (2.10) is true for all integers $\beta$.

Comparing the coefficients of $q^{5 n+i}$ for $i=3,4$ in (3.12), we obtain (2.12). 
The congruence (3.10) is the $\alpha=\beta=0$ case of (2.13). Suppose that the congruence (2.13) holds for $\alpha>0$ with $\beta=0$. From (2.3), the congruence (2.13) with $\beta=0$ becomes

$$
\begin{aligned}
& \sum_{n=0}^{\infty} b\left(16 \cdot 3^{2 \alpha} n+14 \cdot 3^{2 \alpha}+1\right) q^{n} \\
& \quad \equiv 8 f_{6} f_{15}+8 q^{2} f_{15} f_{18}^{3}+8 q^{5} f_{6} f_{45}^{3}+8 q^{7} f_{18}^{3} f_{45}^{3}
\end{aligned}
$$

which implies

$$
\begin{gathered}
\sum_{n=0}^{\infty} b\left(16 \cdot 3^{2 \alpha+1} n+14 \cdot 3^{2 \alpha}+1\right) q^{n} \equiv 8 f_{2} f_{5}, \\
\sum_{n=0}^{\infty} b\left(16 \cdot 3^{2 \alpha+1} n+10 \cdot 3^{2 \alpha+1}+1\right) q^{n} \equiv 8 q^{2} f_{6}^{3} f_{15}^{3}
\end{gathered}
$$

and

$$
\sum_{n=0}^{\infty} b\left(16 \cdot 3^{2 \alpha+1} n+46 \cdot 3^{2 \alpha}+1\right) q^{n} \equiv 8 f_{5} f_{6}^{3}+8 q f_{2} f_{15}^{3} .
$$

Comparing the coefficients of $q^{3 n+2}$ on both sides of the equation (3.16), we get

$$
\sum_{n=0}^{\infty} b\left(16 \cdot 3^{2 \alpha+2} n+14 \cdot 3^{2 \alpha+2}+1\right) q^{n} \equiv 8 f_{2}^{3} f_{5}^{3}
$$

which proves the congruence (2.13) is true for $\alpha+1$ with $\beta=0$. Hence, by induction, the congruence (2.13) is true for any integer $\alpha$ with $\beta=0$. Suppose that the congruence (2.13) holds for some integers $\alpha, \beta>0$.

Employing the equation (3.11) in (2.13) and then comparing the coefficients of $q^{5 n+1}$, we find that

$$
\sum_{n=0}^{\infty} b\left(16 \cdot 3^{2 \alpha} \cdot 5^{2 \beta+1} n+6 \cdot 3^{2 \alpha} \cdot 5^{2 \beta+1}+1\right) q^{n} \equiv 8 q f_{1}^{3} f_{10}^{3}
$$

which proves (2.14). Employing the equation (3.11) in (3.19) and extracting the coefficients of $q^{5 n+4}$, we arrive at

$$
\sum_{n=0}^{\infty} b\left(16 \cdot 3^{2 \alpha} \cdot 5^{2 \beta+2} n+14 \cdot 3^{2 \alpha} \cdot 5^{2 \beta+2}+1\right) q^{n} \equiv 8 f_{2}^{3} f_{5}^{3},
$$

which implies that (2.13) is true for $\beta+1$. Hence, by induction, the congruence (2.13) is true for any non-negative integers $\alpha, \beta>0$.

Employing (2.3) in (2.13) and then comparing the coefficients of $q^{3 n}, q^{3 n+1}$ and $q^{3 n+2}$, we obtain respectively (2.15), (2.17) and (2.18). 
Employing the equation (3.11) in the equation (2.15) and (2.18), we get respectively (2.16) and (2.19).

Using the equations (2.15) and (2.16) along with the equation (3.11), we obtain (2.20) and (2.21) respectively. Using the equations (2.18) and (2.19) along with the equation (3.11), we obtain (2.22) and (2.23) respectively.

Comparing the coefficients of $q^{3 n}$ and $q^{3 n+1}$ in Eq. (2.17), we get (2.24).

Using (2.2) and (2.5) in (3.5), we get

$$
\sum_{n=0}^{\infty} b(8 n+1) q^{n} \equiv 2 \frac{f_{2}^{14} f_{10}^{2}}{f_{1}^{11} f_{4}^{3} f_{5} f_{20}}+8 q \frac{f_{2}^{5} f_{4}^{3} f_{20}}{f_{1}^{8} f_{10}}
$$

and

$$
\sum_{n=0}^{\infty} b(8 n+5) q^{n} \equiv 8 \frac{f_{4}^{5}}{f_{1}^{3} f_{5}}-2 \frac{f_{2}^{5} f_{20}}{f_{1}^{4} f_{4} f_{10}}+8 f_{1}^{3} f_{2}^{2} f_{5} .
$$

Utilizing (3.2) and (3.4) in (3.21), we get

$$
b\left(2^{\alpha+2} n+1\right) \equiv b(4 n+1)
$$

Using (2.2), (2.6) and (2.7) in (3.22), we obtain

$$
\sum_{n=0}^{\infty} b(16 n+5) q^{n} \equiv 8 \frac{f_{2}^{6}}{f_{1} f_{5}}+8 f_{2}^{3}-2 \frac{f_{2} f_{10}}{f_{1} f_{5}}
$$

and

$$
\sum_{n=0}^{\infty} b(16 n+13) q^{n} \equiv 8 f_{8} f_{10}+8 f_{1} f_{2} f_{5}^{3}+8 f_{1}^{3} f_{2}^{5} f_{5} .
$$

Employing (2.7) and (2.8) in (3.24), we get

$$
\sum_{n=0}^{\infty} b(32 n+13) q^{n} \equiv 8 f_{1}^{9}
$$

and

$$
\sum_{n=0}^{\infty} b(32 n+29) q^{n} \equiv 8 f_{1} f_{20}+8 f_{2}^{3} f_{5}^{3} .
$$

The congruence (3.25) is $\alpha=\beta=\gamma=0$ case of (2.25). Suppose that the congruence (2.25) is true for $\alpha \geq 0$ with $\beta=\gamma=0$. From (2.25) with $\beta=\gamma=0$, we arrive at

$$
\sum_{n=0}^{\infty} b\left(32 \cdot 3^{4 \alpha}+12 \cdot 3^{4 \alpha}+1\right) q^{n} \equiv 8 f_{1}^{9}
$$


Using (2.3) in (3.27) and then comparing the coefficients of $q^{3 n}$, we get

$$
\begin{aligned}
& \sum_{n=0}^{\infty} b\left(32 \cdot 3^{4 \alpha+1}+12 \cdot 3^{4 \alpha}+1\right) q^{n} \\
& \quad \equiv 8 f_{1}^{3}+8 q f_{3}^{9} \equiv 8 f_{3}+8 q f_{3}^{9}+8 q f_{9}^{3}
\end{aligned}
$$

which implies

$$
\begin{aligned}
& \sum_{n=0}^{\infty} b\left(32 \cdot 3^{4 \alpha+2}+12 \cdot 3^{4 \alpha+2}+1\right) q^{n} \\
& \quad \equiv 8 f_{1}^{9}+8 f_{3}^{3} \equiv 8 q f_{6} f_{9}^{3}+8 q^{2} f_{3} f_{9}^{6}+8 q^{3} f_{9}^{9}
\end{aligned}
$$

which yields

$$
\sum_{n=0}^{\infty} b\left(32 \cdot 3^{4 \alpha+3}+12 \cdot 3^{4 \alpha+2}+1\right) q^{n} \equiv 8 q f_{3}^{9}
$$

which implies

$$
\sum_{n=0}^{\infty} b\left(32 \cdot 3^{4 \alpha+4}+12 \cdot 3^{4 \alpha+4}+1\right) q^{n} \equiv 8 f_{1}^{9}
$$

which proves the congruence (2.25) is true for $\alpha+1$. By mathematical induction, the congruence (2.25) is true for all integers $\alpha \geq 0$ with $\beta=\gamma=0$. Suppose the congruence (2.25) is true for $\alpha, \beta \geq 0$ with $\gamma=0$. From the equation (2.25) with $\gamma=0$ and then employing (3.11), we get

$$
\sum_{n=0}^{\infty} b\left(32 \cdot 3^{4 \alpha} \cdot 5^{2 \beta+1}+28 \cdot 3^{4 \alpha} \cdot 5^{2 \beta+1}+1\right) q^{n} \equiv 8 q f_{5}^{9}
$$

which implies

$$
\sum_{n=0}^{\infty} b\left(32 \cdot 3^{4 \alpha} \cdot 5^{2 \beta+2}+12 \cdot 3^{4 \alpha} \cdot 5^{2 \beta+2}+1\right) q^{n} \equiv 8 f_{1}^{9}
$$

which proves that the congruence (2.25) with $\gamma=0$ is true for $\beta+1$. So, by induction, the congruence (2.25) with $\gamma=0$ is true for all integers $\alpha, \beta \geq 0$. Suppose that the congruence (2.25) is true for $\alpha, \beta, \gamma \geq 0$ and then utilizing (2.9), we arrive at

$$
\sum_{n=0}^{\infty} b\left(32 \cdot 3^{4 \alpha} \cdot 5^{2 \beta} \cdot 7^{2 \gamma+1} n+4 \cdot 3^{4 \alpha} \cdot 5^{2 \beta+1} \cdot 7^{2 \gamma+1}+1\right) q^{n} \equiv 8 q^{2} f_{7}^{9}
$$


which proves (2.26). Comparing the coefficients of $q^{7 n+2}$ in (3.34), we obtain

$$
\sum_{n=0}^{\infty} b\left(32 \cdot 3^{4 \alpha} \cdot 5^{2 \beta} \cdot 7^{2 \gamma+2} n+12 \cdot 3^{4 \alpha} \cdot 5^{2 \beta} \cdot 7^{2 \gamma+2}+1\right) q^{n} \equiv 8 f_{1}^{9}
$$

which implies that the congruence (2.25) is true for $\gamma+1$. By induction, the congruence (2.25) is true for all integers $\alpha, \beta, \gamma \geq 0$. Using (3.11) in (2.25), we obtain (2.27). The congruence (2.27) implies (2.28). Using (2.3) in (2.25) and then comparing the coefficients of $q^{3 n+1}$ and $q^{3 n+2}$ in the resultant equation, we obtain (2.29) and (2.30) respectively.

Utilizing (3.11) in (2.29) and (2.30), we obtain respectively (2.31) and (2.32).

The congruence (3.26) is $\beta=0$ case of (2.33). Suppose that the congruence (2.33) is true for some integer $\beta \geq 0$. Using (3.11) in (2.33) and then comparing the coefficients of $q^{5 n+1}$, we get

$$
\sum_{n=0}^{\infty} b\left(32 \cdot 5^{2 \beta+1} n+12 \cdot 5^{2 \beta+1}+1\right) q^{n} \equiv 8 f_{4} f_{5}+8 q f_{1}^{3} f_{10}^{3}
$$

which is (2.34). Again using (3.11) in (3.36) and then comparing the coefficients of $q^{5 n+4}$ in the resultant equation, we obtain

$$
\sum_{n=0}^{\infty} b\left(32 \cdot 5^{2 \beta+2} n+28 \cdot 5^{2 \beta+2}+1\right) q^{n} \equiv 8 f_{1} f_{20}+8 f_{2}^{3} f_{5}^{3}
$$

which implies that the congruence (2.33) is true for $\beta+1$. By mathematical induction, the congruence (2.33) is true for all integers $\beta \geq 0$.

Comparing the coefficients of $q^{5 n+i}$ for $i=3,4$ in (2.33) along with (3.11), we obtain (2.35).

From (3.7), we have, modulo 4,

$$
\begin{aligned}
\sum_{n=0}^{\infty} b(8 n+3) q^{n} & \equiv 2 \frac{f_{1} f_{10}}{f_{5}} \\
& \equiv 2 f_{2}^{3}+2 q f_{10}^{3}
\end{aligned}
$$

which reduces to

$$
\sum_{n=0}^{\infty} b(16 n+3) q^{n} \equiv 2 f_{1}^{3}
$$

and

$$
\sum_{n=0}^{\infty} b(16 n+11) q^{n} \equiv 2 f_{5}^{3} .
$$

Equation (3.39) is the $\alpha=\beta=\gamma=0$ case of (2.36). Suppose that the congruence (2.36) is true for $\alpha \geq 0$ with $\beta=\gamma=0$. From (2.36) with $\beta=\gamma=0$, 


$$
\sum_{n=0}^{\infty} b\left(16 \cdot 3^{2 \alpha} n+2 \cdot 3^{2 \alpha}+1\right) q^{n} \equiv 2 f_{1}^{3}
$$

Utilizing (2.3), the equation (3.41) reduces to

$$
\sum_{n=0}^{\infty} b\left(16 \cdot 3^{2 \alpha+1} n+2 \cdot 3^{2 \alpha+2}+1\right) q^{n} \equiv 2 f_{3}^{3}
$$

which yields

$$
\sum_{n=0}^{\infty} b\left(16 \cdot 3^{2 \alpha+2} n+2 \cdot 3^{2 \alpha+2}+1\right) q^{n} \equiv 2 f_{1}^{3}
$$

which implies that the congruence (2.36) is true for $\alpha+1$ with $\beta=\gamma=0$. By mathematical induction, the congruence (2.36) is true for all $\alpha \geq 0$. Suppose that the congruence (2.36) holds for $\alpha, \beta \geq 0$ with $\gamma=0$. Employing (3.11) in (2.36) with $\gamma=0$, we get

$$
\sum_{n=0}^{\infty} b\left(16 \cdot 3^{2 \alpha} \cdot 5^{2 \beta+1} n+2 \cdot 3^{2 \alpha} \cdot 5^{2 \beta+2}+1\right) q^{n} \equiv 2 f_{5}^{3}
$$

which implies

$$
\sum_{n=0}^{\infty} b\left(16 \cdot 3^{2 \alpha} \cdot 5^{2 \beta+2} n+2 \cdot 3^{2 \alpha} \cdot 5^{2 \beta+2}+1\right) q^{n} \equiv 2 f_{1}^{3}
$$

which implies that the congruence (2.36) is true for $\beta+1$ with $\gamma=0$. By mathematical induction, the congruence (2.36) is true for all non-negative integers $\alpha, \beta$ with $\gamma=0$.

Suppose that the congruence (2.36) holds for $\alpha, \beta, \gamma \geq 0$. Employing (2.9) in (2.36), we get

$$
\sum_{n=0}^{\infty} b\left(16 \cdot 3^{2 \alpha} \cdot 5^{2 \beta} \cdot 7^{2 \gamma+1} n+2 \cdot 3^{2 \alpha} \cdot 5^{2 \beta} \cdot 7^{2 \gamma+2}+1\right) q^{n} \equiv 2 f_{7}^{3}
$$

which proves (2.37). The congruence (3.46) reduces to

$$
\sum_{n=0}^{\infty} b\left(16 \cdot 3^{2 \alpha} \cdot 5^{2 \beta} \cdot 7^{2 \gamma+2} n+2 \cdot 3^{2 \alpha} \cdot 5^{2 \beta} \cdot 7^{2 \gamma+2}+1\right) q^{n} \equiv 2 f_{1}^{3},
$$

which implies that the congruence (2.36) is true for $\gamma+1$. By mathematical induction, the congruence (2.36) is true for all integers $\alpha, \beta$ and $\gamma$.

Using (2.3) in (2.36) and comparing the coefficients of $q^{3 n}, q^{3 n+1}$ and $q^{3 n+2}$ respectively, we obtain $(2.38),(2.39)$ and $(2.40)$ respectively.

Comparing the coefficients of $q^{3 n+1}$ and $q^{3 n+2}$ in (2.39), we get (2.41). 
Employing (3.11) in (2.36) and comparing the coefficients of $q^{5 n+3}$, we obtain (2.42).

Comparing the coefficients of $q^{5 n+2}$ and $q^{5 n+4}$ from (2.36) along with (3.11), we obtain (2.43).

Comparing the coefficients of $q^{5 n+i}$ for $i=1,2,3,4$ from (2.42), we arrive at (2.44).

From (3.40), we deduce

$$
\sum_{n=0}^{\infty} b(80 n+11) q^{n} \equiv 2 f_{1}^{3} .
$$

The congruence (3.48) is the $\alpha=\beta=\gamma=0$ case of (2.45). The rest of the proofs of the identities (2.45)-(2.53) are similar to the proofs of the identities (2.36)-(2.44). So, we omit the details.

Employing (2.5) in (3.23), we obtain

$$
\sum_{n=0}^{\infty} b(32 n+5) q^{n} \equiv 2 f_{1}^{3}
$$

and

$$
\sum_{n=0}^{\infty} b(32 n+21) q^{n} \equiv 2 f_{5}^{3} .
$$

The rest of the proofs of the identities (2.54)-(2.71) are similar to the proofs of the identities (2.36)-(2.44). So, we omit the details.

Acknowledgements We would like thank referee for his/her useful comments.

\section{References}

1. Andrews, G.E., Hirschhorn, M.D., Sellers, J.A.: Arithmetic properties of partition with even parts distinct. Ramanujan J. 23(1-3), 169-181 (2010)

2. Berndt, B.C.: Ramanujan's Notebooks, Part III. Springer, New York (1991)

3. Calkin, N., Drake, N., James, K., Law, S., Lee, P., Penniston, D., Radder, J.: Divisibility properties of the 5-regular and 13-regular partition functions. Integers 8, A60 (2008)

4. Chen, S.-C.: On the number of partition with distinct even parts. Discrete Math. 311(12), 940-943 (2011)

5. Cui, S.P., Gu, N.S.S.: Arithmetic properties of $\ell$-regular partitions. Adv. Appl. Math. 51, 507-523 (2013)

6. Hirschhorn, M.D.: Ramanujan's “most beautiful identity". Am. Math. Mon. 118, 839-845 (2011)

7. Hirschhorn, M.D., Sellers, J.A.: Elementary proofs of parity results for 5-regular partitions. Bull. Aust. Math. Soc. 81, 58-63 (2010)

8. Lin, B.L.S.: Arithmetic properties of bipartitions with even parts distinct. Ramanujan J. 33(2), 269-279 (2014)

9. Mahadeva Naika, M.S., Hemanthkumar, B.: Arithmetic properties of 5-regular bipartitions. Int. J. Number Theory 13(4), 937-956 (2017)

10. Mahadeva Naika, M.S., Hemanthkumar, B., Bharadwaj, H.S.S.: Color partition identities arising from Ramanujan's theta functions. Acta Math. Vietnam 41(4), 633-660 (2016) 
11. Merca, M.: New relations for the number of partitions with distinct even parts. J. Number Theory 176, $1-12$ (2017)

12. Watson, G.N.: Theorems stated by Ramanujan (VII): theorems on continued fractions. J. Lond. Math. Soc. 4, 39-48 (1929) 